\title{
Energy splitting between triplet and singlet exciplex states determined with E-type delayed fluorescence
}

\author{
Bernd Frederichs, Hubert Staerk* \\ Max-Planck-Institut für biophysikalische Chemie, Abt. Spektroskopie und photochemische Kinetik, 37070 Göttingen, Germany
}

\section{A R T I C L E I N F O}

\section{Article history:}

Received 29 April 2008

In final form 26 May 2008

Available online 17 June 2008

\begin{abstract}
A B S T R A C T
In the redox system with $N, N$-diethylaniline as electron donor and 1,4-dicyanobenzene as electron acceptor the singlet and triplet exciplex states in the nonpolar solvent methylcyclohexane are almost degenerate. The lowest excited state is the triplet exciplex state from which the singlet exciplex state is populated in a fast endothermic reaction. The energy splitting has been determined experimentally as $\Delta E \approx 0.1 \mathrm{eV}$ via E-type delayed fluorescence.
\end{abstract}

(C) 2008 Elsevier B.V. All rights reserved.

\section{Introduction}

The first experimental evidence of molecular complex formation in the excited state between two different components of a redox system leading to a so-called hetero excimer was given already in 1961 [1,2]. Since then a lot of research work has been done on numerous examples of complex formation in the lowest electronically excited singlet state. Studies of complex formation in the lowest excited triplet state, however, have been very scarce. This is because generally phosphorescence can be observed in rigid media only where diffusion is extremely slow. Hence exciplex phosphorescence was studied only with complexes which are stable in the ground state [3]. Later, Beens and Weller [4,5] attempted a classification of charge transfer (CT) systems and arrived at the general rule for necessary conditions of exciplex formation on the grounds of the redox properties of electron donor (D) and acceptor (A), ionization potential (IP) and electron affinity (EA):

$\mathrm{IP}_{\mathrm{A}}-\mathrm{IP}_{\mathrm{D}}>0$ and $\mathrm{EA}_{\mathrm{A}}-\mathrm{EA}_{\mathrm{D}}>0$

This requires that

$\mathrm{IP}_{\mathrm{A}}+\mathrm{EA}_{\mathrm{A}}>\mathrm{IP}_{\mathrm{D}}+\mathrm{EA} \mathrm{A}_{\mathrm{D}}$

i.e., the sum (IP + EA) of a molecular pair determines the direction of the electron transfer. From the redox data and singlet and triplet energy data of the system $N, N$-diethylaniline (DEA = D) and 1,4-dicyanobenzene $(\mathrm{DCNB}=\mathrm{A})$ it could be shown that, in the inert solvent methylcyclohexane $(\mathrm{MCH})$, the triplet $\mathrm{CT}$ complex is formed from the triplet excited state of the donor, ${ }^{3} \mathrm{D}^{*}$, and the acceptor $\mathrm{A}$ (at low concentration) in its ground state, according to the reaction

${ }^{3} \mathrm{D}^{*}+\mathrm{A} \stackrel{k_{c} c_{A}}{\rightarrow}\left(\mathrm{D}^{+} \mathrm{A}^{-}\right)$

\footnotetext{
* Corresponding author. Fax: +49551 2011501

E-mail address: hstaerk@gwdg.de (H. Staerk).
}

Furthermore, it was assumed [4,5] that the observed CT emission could only arise from the $\mathrm{CT}$ singlet state which is populated due to the endothermic reaction

${ }^{3}\left(D^{+} A^{-}\right) \rightarrow{ }^{1}\left(D^{+} A^{-}\right)$

observable via E-type delayed fluorescence [6,7].

The purpose of the present work was to extend the measurements of this system in order to verify the proposed $[4,5]$ reaction mechanism and to obtain parameters which allow the determination of the energy splitting $\Delta E$ between the exciplex triplet and exciplex singlet state.

\section{Experimental}

$N, N$-Diethylaniline (DEA), purissinum Fluka, was redestilled under nitrogen atmosphere in the dark. 1,4-Dicianobenzene (DCNB) was from Fluka. The water-free methylcyclohexane $(\mathrm{MCH}$, through molecular sieve $4 \AA$, $2 \mathrm{~mm}$ ) solutions were kept under nitrogen atmosphere during sample preparation and measurements. Six freeze-pump-thaw cycles of a vacuum degassing process were applied up to a final stationary pressure of $5 \times 10^{-6}$ Torr before sealing the quartz cuvettes. The versatile spectrometer equipped with a cryostat has been described elsewhere [8]. The excitation light (313 nm from an OSRAM 150 Watt mercury high pressure lamp) was chopped with $50 \mathrm{rpm}$ for the quasi photostationary intensity measurements and with 3000 rpm for the lifetime measurements. The signals from the photomultiplier were measured with a PAR/HR-8 lock-in amplifier and PAR/TDH-9 multichannel analyzer, respectively. It turned out to be of utmost importance for this study around and below zero degrees centigrade that samples are free of traces of water. Otherwise stray light effects during spectroscopic measurements would lead to erroneous results. 


\section{Results and discussion}

Emission spectra of deaerated solutions are shown in Fig. 1. The donor (D) is primarily excited. The intensity $I$ of its fluorescence with maximum at $338 \mathrm{~nm}$ remains unaltered with varying acceptor concentration, while the intensity $I^{\prime}$ of the blue-green emission with maximum at $489 \mathrm{~nm}$ increases with increasing acceptor concentration $c_{\mathrm{A}}$. This emission can be strongly quenched by oxygen and by biphenyl whose triplet energy at $2.92 \mathrm{eV}$ is below that of diethylaniline with $3.23 \mathrm{eV}$. Lifetime measurements of the bluegreen emission give values of about $10^{-5} \mathrm{~s}$ at room temperature, which shows the delayed character of this fluorescence. For $c_{\mathrm{A}}$-concentrations $2 \times 10^{-5} \mathrm{M}$ and $5 \times 10^{-6} \mathrm{M}$ exponential decay times of the delayed luminescence as a function of temperature are shown in Fig. 2. These observations corroberate a reaction mechanism as shown in the scheme in Fig. 3.

The lifetime of the prompt fluorescence from ${ }^{1} \mathrm{D}^{*}$, $\tau_{\mathrm{F}}=\left(k_{\mathrm{f}}+k_{\mathrm{d}}+\mathrm{k}_{\mathrm{isc}}\right)^{-1} \approx 4 \mathrm{~ns}$, is independent of the presence of the acceptor at the low concentrations used. The triplet lifetime of the donor $\left({ }^{3} \mathrm{D}^{*}\right)$ at zero acceptor concentration is $\tau_{\mathrm{T}}=\left(k_{\mathrm{p}}+k_{\mathrm{n}}\right)^{-1}$

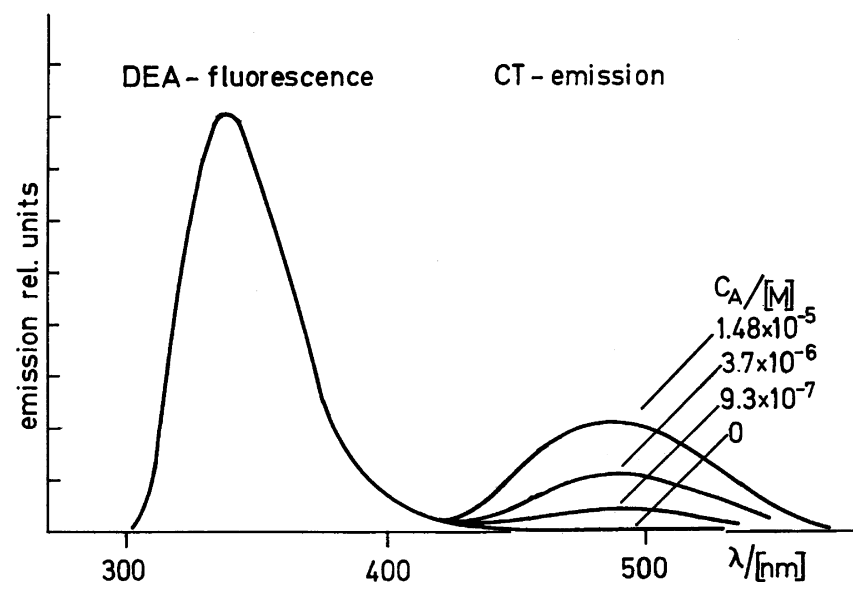

Fig. 1. Emission spectra (corrected) of deaerated solutions of $2 \times 10^{-4} \mathrm{M} \mathrm{N}, N$ diethylaniline $\left(\mathrm{DEA}=\right.$ donor) and different conscentrations $c_{\mathrm{A}}$ of 1.4-dicyanobenzene $(\mathrm{DCNB}=$ acceptor $)$ in methylcyclohexane $(\mathrm{MCH})$ at room temperature. Excitation $313 \mathrm{~nm}\left({ }^{1} \mathrm{D}^{*}\right)$.

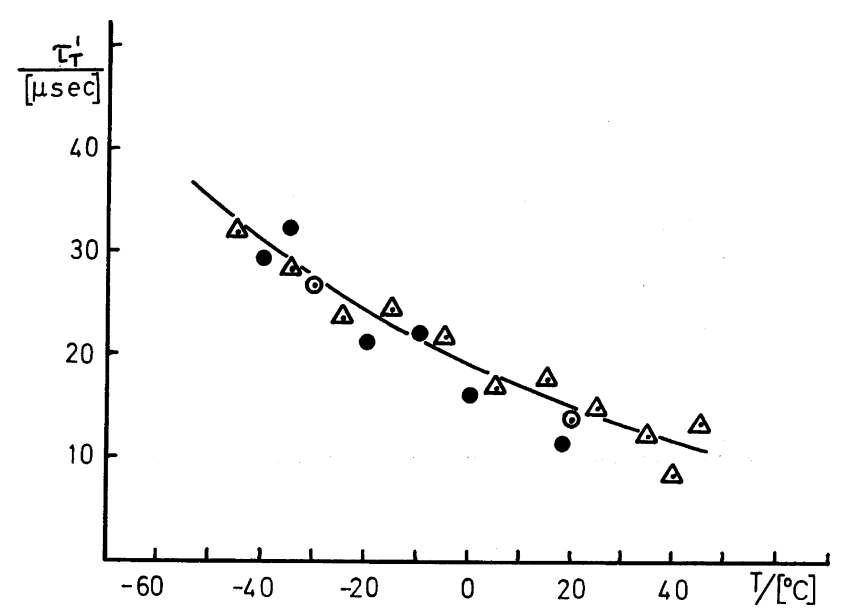

Fig. 2. Temperature dependence of decay times $\tau_{T}^{\prime}(T)$ of delayed emission of the $\mathrm{DEA} / \mathrm{DCNB}$ exciplex in $\mathrm{MCH}$ at $489 \mathrm{~nm}$; from two samples with $c_{\mathrm{A}}$-concentrations $2 \times 10^{-5} \mathrm{M}$ (open circles, decreasing temperatures; closed circles, increasing temperature) and $5 \times 10^{-6} \mathrm{M}$ (triangles, decreasing temperatures). A concentration dependence was not detectable.

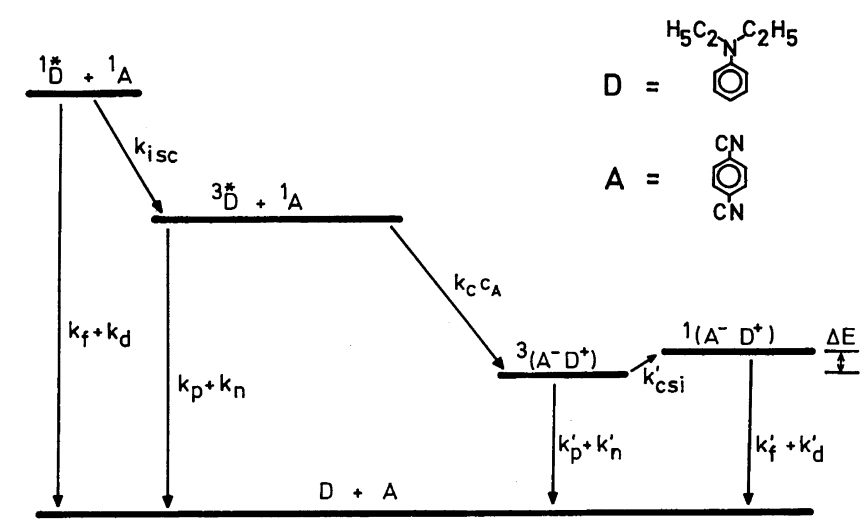

Fig. 3. Overall reaction scheme for the formation of the triplet exciplex ${ }^{3}\left(A^{-} D^{+}\right)$and its subsequent deactivation by the mechanism of E-type delayed emission. Primed letters pertain to reaction parameters which originate from a complex state.

which has been determined in a separate absorption experiment as $9.3 \times 10^{-6} \mathrm{~s}$ at room temperature.

With this result and $I^{\prime}\left(c_{\mathrm{A}}\right)$ at $489 \mathrm{~nm}$ from Fig. 1 the complementary Stern-Volmer equation

$\frac{1}{I^{\prime}\left(c_{\mathrm{A}}\right)}=\frac{1}{I^{\prime}(\infty)}\left(1+c_{\mathrm{h}} / c_{\mathrm{A}}\right)$

with the half-value concentration

$c_{\mathrm{h}}=\left(k_{\mathrm{c}} \tau_{\mathrm{T}}\right)^{-1}$

can be evaluated. By plotting $1 / I^{\prime}\left(c_{\mathrm{A}}\right)$ versus $1 / c_{\mathrm{A}}$ one obtains $I^{\prime}(\infty)$ the (extrapolated) intensity at $c_{\mathrm{A}}=\infty$ and $c_{\mathrm{h}}=6 \times 10^{-6} \mathrm{M}$. Finally, Eq. (6) gives the bimolecular rate constant for the diffusion controlled triplet exciplex formation (cf. Fig. 3) $k_{\mathrm{c}}=18 \times 10^{9} \mathrm{M}^{-1} \mathrm{~s}^{-1}$.

As a proof for the assumption that, with the chosen CT-pair, the singlet and triplet exciplex levels are almost degenerate, a rather simple relation is derived in the following, which can be evaluated graphically.

From the reaction scheme in Fig. 3 the overall quantum efficiency $\varphi^{\prime}$ is obtained for the emission from the ${ }^{1}\left(\mathrm{~A}^{-} \mathrm{D}^{+}\right)$state as the product of all consecutive quantum efficiencies

$\varphi^{\prime}=\varphi_{\text {isc }} \times \varphi_{\mathrm{c}} \times \varphi_{\mathrm{csi}}^{\prime} \times \varphi_{\mathrm{f}}^{\prime}$

with

$\varphi_{\mathrm{isc}}=k_{\mathrm{isc}} \tau_{\mathrm{f}}$

$\varphi_{\mathrm{c}}=\frac{k_{\mathrm{c}} \tau_{\mathrm{T}} c_{\mathrm{A}}}{1+k_{\mathrm{c}} \tau_{\mathrm{T}} c_{\mathrm{A}}}$

$\varphi_{\mathrm{csi}}^{\prime}=\frac{k_{\mathrm{csi}}^{\prime}}{k_{\mathrm{p}}^{\prime}+k_{\mathrm{n}}^{\prime}+k_{\mathrm{csi}}^{\prime}}$

$\varphi_{\mathrm{f}}^{\prime}=\frac{k_{\mathrm{f}}}{k_{\mathrm{f}}^{\prime}+k_{\mathrm{d}}^{\prime}+k_{\mathrm{ics}}^{\prime}}$

At the limit of infinite acceptor concentration $\left(c_{\mathrm{A}} \rightarrow \infty\right), \varphi_{\mathrm{c}}$ approaches the value one and with the exciplex triplet lifetime $\tau_{\mathrm{T}}^{\prime}=\left(k_{\mathrm{p}}^{\prime}+k_{\mathrm{n}}^{\prime}+k_{\mathrm{csi}}^{\prime}\right)^{-1}$ we obtain

$\varphi_{\infty}^{\prime}=\varphi_{\text {isc }} \varphi_{\mathrm{f}}^{\prime} k_{\mathrm{csi}}^{\prime} \tau_{\mathrm{T}}^{\prime} \propto I_{\infty}^{\prime}$

$k_{\mathrm{csi}}^{\prime}$ may be treated in analogy to the classical example of E-type delayed fluorescence [6,7]. This process corresponds to a thermal activation into a higher vibrational level of the triplet CT state, followed by intersystem crossing into the singlet level of the CT complex. One can write

$k_{\mathrm{csi}}^{\prime}(T)=\frac{k_{\mathrm{isc}}^{\prime}}{3} \exp \left(-\frac{\Delta E}{R T}\right)$ 


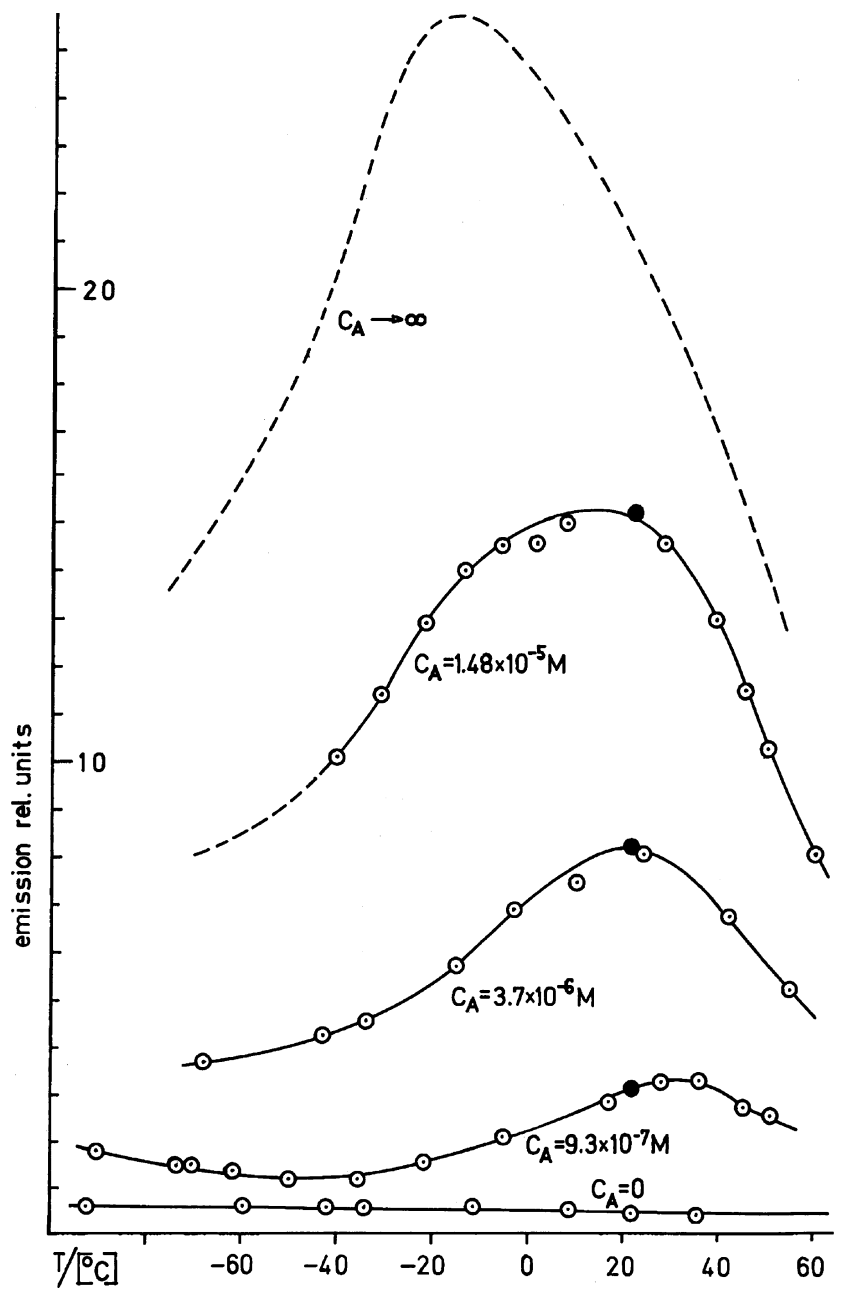

Fig. 4. Delayed exciplex emission intensity $I^{\prime}$ plotted as a function of temperature for three acceptor (DCNB) concentrations $c_{\mathrm{A}}$. Donor concentration $c_{\mathrm{D}}=2.0 \times 10^{-4} \mathrm{M}$.

where $k_{\text {isc }}^{\prime}$ is the rate constant of the radiationless process ${ }^{3}\left(\mathrm{~A}^{-} \mathrm{D}^{+}\right) \leftarrow{ }^{1}\left(\mathrm{~A}^{-} \mathrm{D}^{+}\right)$and $\Delta E$ is the corresponding singlet-triplet energy splitting. The multiplicity difference is taken into account by the factor $1 / 3$. Finally, we obtain with Eq. (12) the following temperature dependent relation:

$\frac{I_{\infty}^{\prime}(T)}{\tau_{\mathrm{T}}^{\prime}} \propto \varphi_{\mathrm{isc}} \varphi_{\mathrm{f}}^{\prime} \frac{k_{\mathrm{isc}}^{\prime}}{3} \exp \left(-\frac{\Delta E}{R T}\right)$

$I_{\infty}^{\prime}(T)$ is obtained from the set of experimental data shown in Fig. 4 where the delayed exciplex emission intensity, $I^{\prime}$, is plotted as a function of temperature for three acceptor (DCNB) concentrations $\left(c_{\mathrm{A}}\right)$; cf. Eq. (3).

Thus, a semilogarithmic plot of Eq. (14), i.e., $\log I_{\infty}^{\prime} / \tau_{\mathrm{T}}^{\prime}$ versus $1 /$ $T$, with $\tau_{\mathrm{T}}^{\prime}$ from Fig. 2, yields the curve shown in Fig. 5. Its shape is determined by $\Delta E$ and the temperature dependence of $\log \varphi_{\text {isc }} \varphi_{\mathrm{f}}^{\prime}$. However, whereas at high temperature it turns out that

$\partial \log \varphi_{\mathrm{isc}} \varphi_{\mathrm{f}}^{\prime} / \partial 1 / T>0$,

approaching zero with decreasing temperature, one obtains at lower temperature from the slope in Fig. 5 a lower limiting value

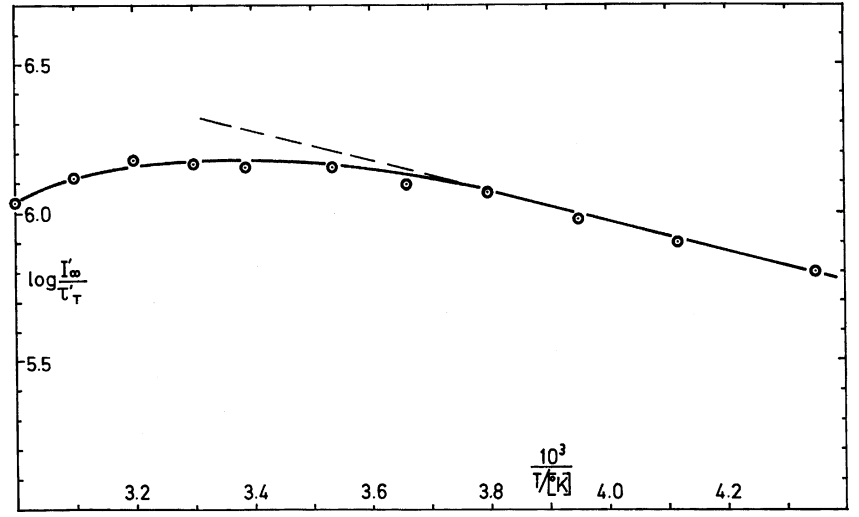

Fig. 5. Eq. (14) plotted versus $1 / T . \Delta E \approx 0.1 \mathrm{eV}$ is obtained from the slope at lower temperature.

$\Delta E>2.3 \mathrm{kcal} / \mathrm{Mol} \approx 0.1 \mathrm{eV}$ ( $4 \mathrm{kT}$ at room temperature), for the energy difference between the singlet and triplet exciplex levels of the system DEA/DCNB in MCH.

The occurrence of E-type delayed emission from this CT complex made it possible to study CT complex formation in the triplet state by emission spectroscopy.

The same system, DEA + DCNB, however, in a polar solvent, e.g. acetonitrile, would be particularly suitable to further extend the studies, with interesting aspects towards the generation of radical ion pairs in the overall triplet and singlet spin state $-{ }^{3,1}\left({ }^{2} \mathrm{~A}^{-}+{ }^{2} \mathrm{D}^{+}\right)$ are then the lowest states above the ground state - and the observation of magnetic field effects of the type described earlier ([9] and references therein).

The lifetime in acetonitrile of the primarily excited electron donor DEA is only a few nanoseconds. Fluorescence quencher concentrations of the order of $10^{-5} \mathrm{M}$ or lower would therefore not produce radical ion pairs in the overall singlet spin state in a significant amount but primarily and predominantly in the triplet state. The change of spin multiplicity of the initial triplet radical ion pair that originates from the hyperfine coupling between electron spins and nuclear spins within each radical ion leads to singlet radical ion pairs, with possible consecutive association to a radiative singlet exciplex (as we have shown with other systems [9]). The yield of singlet products generated from radical ion pairs in the triplet spin state in a geminate recombination is expected to decrease (due to the Zeeman effect) to about $30-50 \%$ by an external magnetic field of only 10-50 mT, compared to its zero field value. Such effects are particularly pronounced in systems where donor and acceptor are linked by a long aliphatic chain (e.g. $\left.\left(\mathrm{CH}_{2}\right)_{16-23}\right)$ where homogeneous recombination reactions of the radical ion pair species are absent [9].

\section{References}

[1] H. Leonhardt, A. Weller, Z. Phys. Chem. N.F. 29 (1961) 277.

[2] H. Leonhardt, A. Weller, Ber. Bunsenges. Physik. Chem. 63 (1963) 791.

[3] S. Iwata, J. Tanaka, S. Nagakura, J. Chem. Phys. 47 (1967) 2203.

[4] H. Beens, A. Weller, in: E.C. Lim (Ed.), Molecular Luminescence, Benjamin, New York, 1969, p. 203.

[5] H. Beens, A. Weller, Acta Phys. Pol. 34 (1968) 539.

[6] C.A. Parker, C.G. Hatchard, Trans. Faraday Soc. 57 (1961) 1894

[7] C.A. Parker, C.G. Hatchard, Trans. Faraday Soc. 59 (1963) 284.

[8] H. Staerk, J. Lumin. 11 (1976) 413.

[9] H. Staerk, H.-G. Busmann, W. Kühnle, R. Treichel, J. Phys. Chem. 95 (1991) 1906. 